\title{
Therapeutic Mammoplasty: Is it a Solution for Treatment of Early Breast Cancer?
}

\author{
MAHMOUD R. SHEHATA, M.D.*; MOHAMED A.M. RIZK, M.Sc.*; HESHAM F. FOUAD, M.Sc.*; \\ TAREK A. MOSTAFA, M.D.* and YOUSSEF HASSAN, M.D.** \\ The Departments of General Surgery* and Plastic \& Reconstructive Surgery**, Faculty of Medicine, Assiut University, \\ Assiut, Egypt
}

\begin{abstract}
Conservative breast surgery has become the standard treatment of early stages breast cancer as it provides the same oncological safety as the classic mastectomy, but in many cases, this led to marked breast deformity especially when the resected mass is large or the mass is located in difficult areas as the upper inner breast quadrant.

Objective: This study aims to evaluate the oncological and aesthetic outcome of the therapeutic mammoplasty in treating breast cancers located in the upper breast quadrants.

Patients and Methods: Oncoplastic excision of breast cancer by doing bilateral inferior pedicle mammoplasty was performed on 50 patients in Assiut University Hospital between 2016 and 2020. This study was performed on patients with early-stage breast cancer located in the upper inner or outer breast quadrants including those who received neoadjuvant chemotherapy. Evaluation of the short-term surgical outcomes including hospital stay, complications, and symmetrization was done. Patient satisfaction and recurrence were also assessed.
\end{abstract}

Results: A total of 50 patients were included in the study. The mean age was 48.7 years and the tumors were located in the upper outer quadrant, upper inner quadrant, and upper breast pole in 35,8 , and 7 patients respectively. The mean operative time was 3.2 hours. Only $12 \%$ of patients showed early complications in form of seroma and wound dehiscence. $90 \%$ of the patients showed good symmetry and only $10 \%$ showed fair symmetry in the last follow-up (18 months postoperative). $94 \%$ of our patients were highly satisfied with the aesthetic outcome. We didn't report any recurrence.

Conclusion: This study shows that oncoplastic breast surgery, as the therapeutic mammoplasty, for breast cancers in the upper breast quadrants can achieve equivalent oncological results as the classic breast conservative surgery with advantageous cosmetic outcome and breasts symmetry especially the inferior pedicle mammoplasty technique which led to much better patients' satisfaction than the conventional conservative surgeries.

Key Words: Mammoplasty - Cancer - Breast.

\section{INTRODUCTION}

Since the beginning of recorded time, the breast has been a symbol of motherhood, femininity, and sexuality. It has been portrayed throughout history in works of art symbolizing each of these aspects of a woman's life [1].

Worldwide breast neoplasm is the most common cancer in females contributing by about $29 \%$ of cancers in women [2]. In Egypt this problem is more pronounced as it represents $32.04 \%$ of female cancers. This problem is aggravated by the late presentation of most of these females as $49.7 \%$ of them have regional spread at the time of presentation and $11.9 \%$ of them have distant metastasis. But now thanks to the screening programs and females awareness higher percentage of females diagnosed at early stages and became candidates for oncoplastic breast surgery [3].

It is well known that the upper outer breast quadrant is the most common site of breast cancer due to condensation of the breast tissue, also it is known that the upper inner quadrant (known as the no man's land) is the most cosmetically sensitive area even with minor resections as it has less mammary tissue [4].

Considering the established goals of breast cancer surgery is to resect cancer with complete oncological safety but it also showed a great evolution over years to get the optimal oncological result with a much higher aesthetic satisfaction [1].

As long as the oncological outcome is not affected, the increasing interest in the aesthetic outcome and the symmetrization of both breasts have led to the new era of breast conservative surgery (BCS) then oncoplastic breast surgery (OPS). In 1998 Audretsch et al., invented the term oncoplastic surgery describing the new techniques for resecting larger breast cancers with an adequate safety margin and the immediate use of local tissue rearrangement or tissue displacement to cover the 
resulting defects with better aesthetic outcome $[2,3]$.

The basic four components of OPS are adequate tumor resection, appropriate partial reconstruction after tumor excision, complete reconstruction for mastectomy, and symmetrization of the other breast [4].

It's known that $10-12 \%$ of the breast tissue is the maximum amount that can be excised during BCT otherwise the cosmetic outcome won't be satisfactory especially for large masses located in the upper outer or innerquadrants of the breast. The new oncoplastic techniques especially the more sophisticated ones as therapeutic mammoplasty (as the inferior mammoplasty used in this study) allow resection of up to 200-500g or from more difficult tumor locations as the upper inner quadrantor the upper pole of the breast $[\mathbf{5 , 6 ]}$.

Also, the classic BCS techniques led to considerable asymmetry and the marked deviation of the nipple-areola complex (NAC). The well-established pedicle mammoplasty techniques done by plastic surgeons can reliably keep the nipple-areola complex well perfused in any breast of almost any size and shape which allows resections of larger masses and at a longer distance from the (NAC) and the added advantage of bilateral breast reduction $[7,8]$.

All this mandates the use of the therapeutic mammoplasty technique used in this study (inferior pedicle mammoplasty) by adding the experience of plastic surgeons and oncosurgeons to get the best oncological safety with highly satisfying breast shape and symmetry.

\section{PATIENTS AND METHODS}

This study aimed to find the rule of therapeutic mammoplasty in treatment of early breast cancer by doing therapeutic inferior pedicle mammoplasty with contralateral breast reduction to obtain proper symmetry and was performed on 50 female patients as a collaboration between general surgery and plastic surgery doctors who presented with operable breast cancer located in the upper breast quadrants and greater than $20 \%$ of the breast volume. They were admitted to Assiut University Hospitals, Egypt between Jan 2016 and May 2020. The tumors were confirmed histologically and staged according to TNM staging, $7^{\text {th }}$ edition, American Joint Committee of Cancer staging system (Edge SB, 2010).

\section{Inclusion criteria:}

- Female patients aging from 20 to 65 years.

- Breast cancer that was confirmed histopatholog- ically and the malignancy staging was carcinoma in situ (CIS) or invasive up to stage IIB either primary or after neoadjuvant therapy.

- Patients who had a large mass to breast ratio (exceeding 20\%).

- Mass was located in the upper outer breast quadrant, the upper pole of the breast (12 o'clock), or the upper inner quadrant close or away from the nipple-areola complex.

- Average to large breast size and mild, moderate, or marked degree of ptosis.

- The most important characteristic of these patients was that they were willing to have bilateral breast surgery to have the advantage of breast symmetrization.

\section{Exclusion criteria:}

- Female patients with age younger than 20 years or older than 65 years.

- Advanced primary breast cancer of stage III or higher.

- Tumors located in the lower half of the breast or had Nipple or areola involvement.

- Patients with any contraindication of BCS as multicentric lesions, recurrent malignancy, or previous breast irradiation.

- Female patients were unwilling for BCS or bilateral breast operations.

Patients in this study underwent bilateral inferior pedicle mammoplasty to excise the cancer with safety margin and contralateral breast reduction mammoplasty and followed-up for 18 months postoperative to detect early complications, aesthetic outcome, breasts symmetry, and recurrence.

\section{Multidisciplinary team (MDT):}

(MDT) included oncosurgeons, plastic surgeons, radiologist, oncologist, and pathology doctor reviewed every single case independently, and reached the final optimal decision for every patient.

\section{Patient counseling and consent:}

Operative details of the surgical technique to be used were explained for every patient taking into consideration the best option for every patient based on MDT decision, size of the breast and tumor, location of the tumor, and the patient acceptance of bilateral breast surgery.

Formal consent was signed by the patients after counseling and informing them of the postoperative treatment (adjuvant therapy), the possible complications, and the expected aesthetic outcome. 


\section{Medical photography:}

The need and the way of medical photographs were discussed with the patients and consented to it. Patients were photographed in (P.A, RT oblique, LT oblique, RT lateral, and LT lateral) positions.

Surgical technique:

\section{Inferior pedicle mammoplasty:}

This approach was done on both breasts during the same operative session.

Preoperative marking was done while the patient in an upright standing position bilaterally by allocation of the tumor on the skin surface of the affected breast, a central line was drawn from the suprasternal notch to the umbilicus, another two vertical lines were drawn bilaterally from the midclavicular point to the nipple and continued to the inframammary fold. The desired location of the future nipple was designed based on the inframammary fold, an inverted V shape was drawn with its apex pointing to the future nipple and two horizontal lines were drawn from each end of the inverted $\mathrm{V}$ shape and extended laterally to the inframammary fold. The vascular pedicle of the breast mound, which supports the parenchyma and nipple-areolar complex, was based inferiorly and the pedicle base width was 6-12cm (Fig. 1) [9]. The skin was incised around the drawn lines to allow excision of the tumor with a safety margin from the affected breast and similar amount of breast parenchyma from the other side. The skin overlying the cancer was then undermined corresponding to the mammoplasty fashioned and excision of the mass with safety margin all around and down to the pectoralis major muscle was done. Marking of every margin by stitches was done and sent for frozen section histopathology to consider if further excision was needed. Surgical clips were placed in the tumor bed to facilitate the proper delivery of postoperative radiotherapy.

Intraoperative frozen section pathology evaluation of the resected masses considered 5-mm surgical margin as the cut-off point for negative margins.

An ipsilateral axillary evacuation was done for all patients from a separate axillary incision.

Contralateral breast reduction was done for the other breast to reach the desired breast symmetrization. (Fig. 2A,B,C) (Fig. 3A,B).

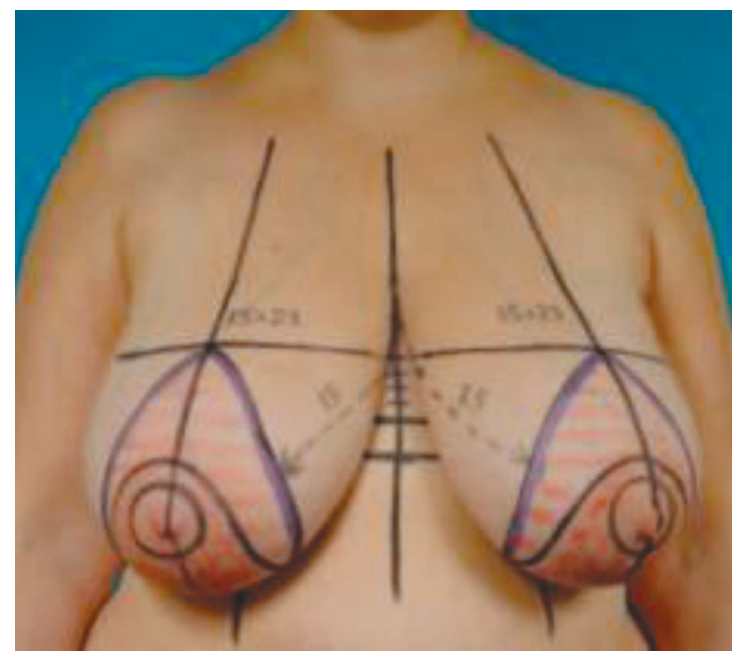

Fig. (1): Pre-operative marking of inferior pedicle mammoplasty [9].

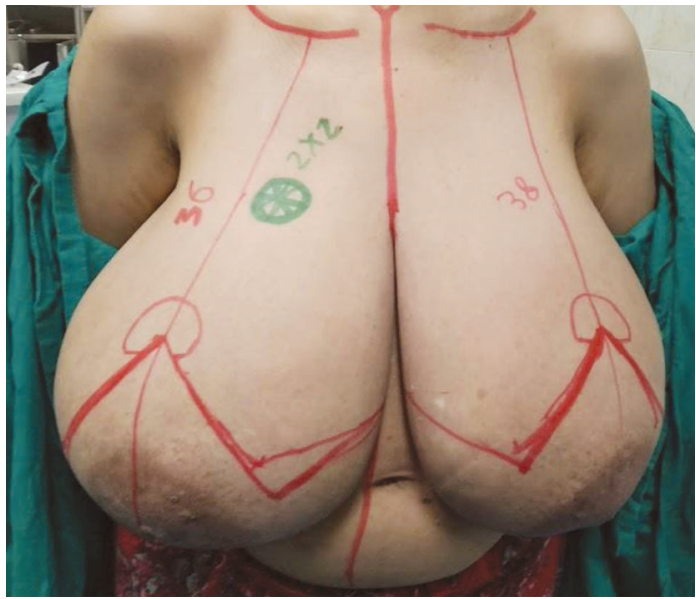

Fig. (2A): Pre-operative marking of inferior pedicle mammoplasty in female patient with RT. Upper inner quadrant breast mass.
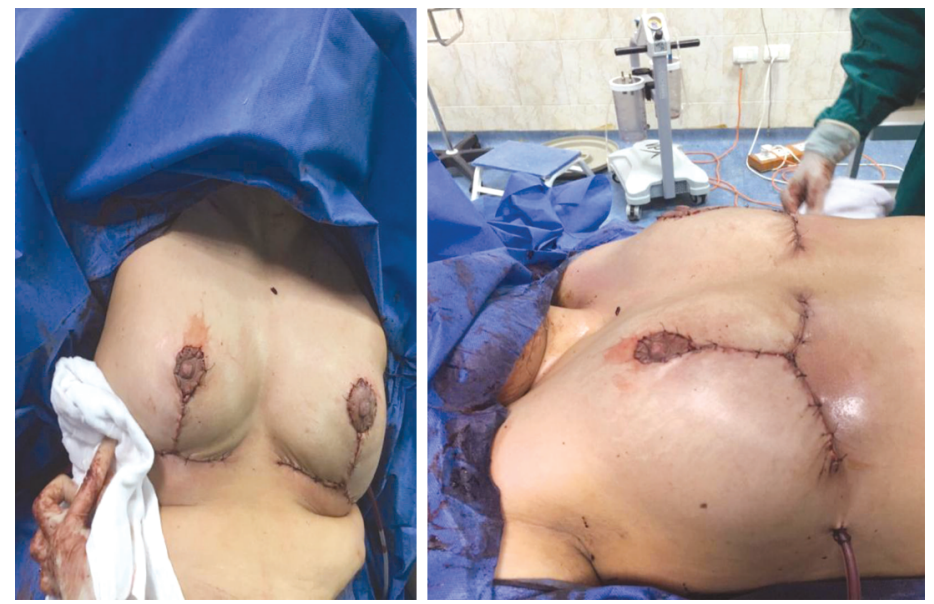

Fig. (2B): Immediate post-operative result. 

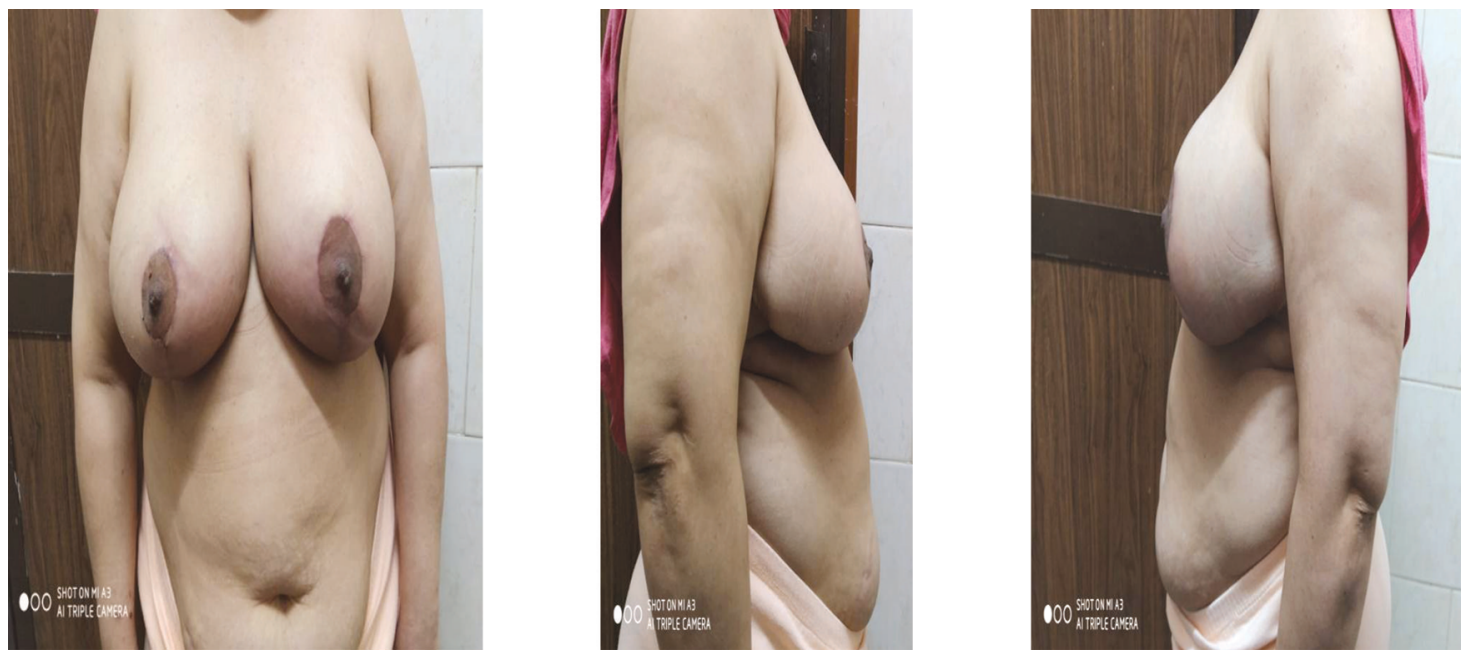

Fig. (2C): Late post-operative result.
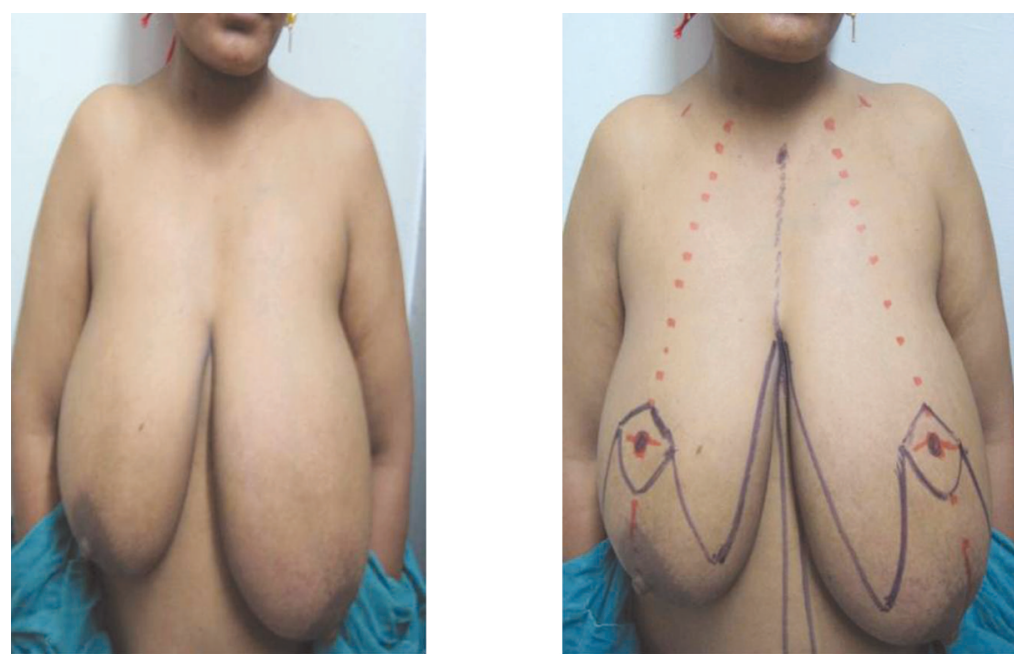

Fig. (3A): Pre-operative marking of inferior pedicle mammoplasty in female patient with LT. Upper outer quadrant breast mass with breast asymmetry.
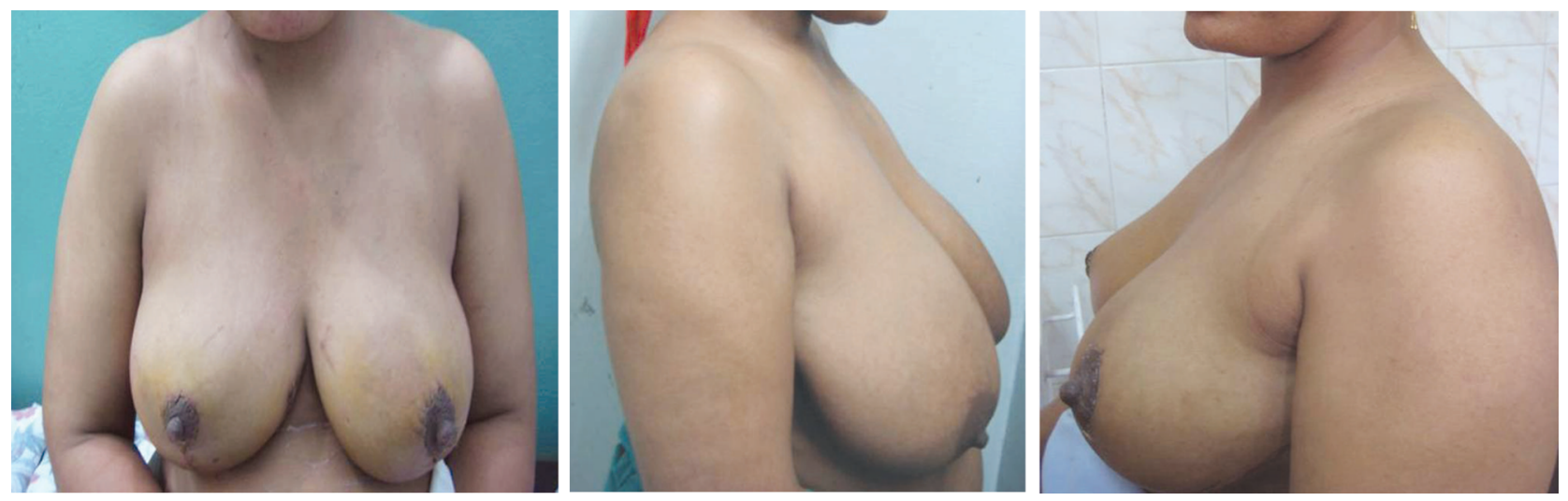

Fig. (3B): Late post-operative result.

\section{Intraoperative:}

Patients were evaluated for surgical time, amount of blood loss, and breast symmetrization.
Post-operative care:

For all operated patients the following was done: - All patients were kept in the hospital overnight until adequate oral intake was attained. 
- Patients were taught to move the ipsilateral arm gradually.

- All patients had the drains removed when they yielded less than $30 \mathrm{cc} / 24$ hours.

- The sutures were removed on day 10 postoperatively.

All patients underwent breast radiation therapy. Also, the other adjuvant lines as chemotherapy, hormonal and targeted therapy as decided by consultants of medical oncology as recommended by the standard protocols.

Follow-up of patients:

Patients in each of the groups mentioned earlier were evaluated as regards the:

- Oncological and surgical outcome.

- Aesthetic outcome.

- The patient's satisfaction.

Early post-operative period (during the first visit 10 days after the operation):

- Viability of the nipple-areola complex on both sides.

- Wound outcome; wound dehiscence or wound infection.

- Presence of seroma in the operative site.

- Presence of fat or skin necrosis.

Late post-operative period (during the second visit one month after the operation):

- The symmetry of the breast and nipple and patient's satisfaction.

- Delayed wound complications; scar hypertrophy or keloid formation. and patient satisfaction.
Last visit (eighteen months after the operation):

- Local recurrence.

- The symmetry of the breast and nipple.

- Patient satisfaction.

All patients were evaluated for local recurrence, and metastasis every 3 months for 1 year and then after 6 months.

Follow-up was done by physical examination, sonomammography or breast MRI if local recurrence was suspected, pelviabdominal ultrasound, computed tomography (CT) chest, CT brain, bone scan.

\section{Cosmetic outcomes are classified into:}

- Good: Where the symmetry of size and shape of both breasts is not affected, minimal complications, and patient satisfied with the result.

- Fair: Where the symmetry of both size and shape of breasts is mildly affected, moderate complications and patient satisfied with the result as compared with modified radical mastectomy.

- Poor: Where the symmetry of both size and shape of breasts is grossly affected, presence of complications or patient was not satisfied with the result.

The aesthetic outcome was evaluated by two blind consultant doctors and a patients' questionnaire used by Eichlier et al., (answer and analysis) (Table 1) [10].

\section{Statistical analysis:}

Data analyses were conducted using SPSS 22 (IBM, SPSS Statistics). Data were statistically described in terms of range, mean, and percentage when appropriate.

Table (1): List of questions of all evaluated parameters as described by Eichlier et al., [9].

1 Evaluate the overall cosmetic outcome of your breast

- On a scale from 1 to 5

(1: very satisfied, 5 : very unsatisfied

2 Are you satisfied with the appearance and amount of scar tissue?

3 Do you like the current shape of the breast?

4 Are you currently satisfied with the appearance of the breast?

5 Are you currently satisfied with the size of the breast?

6 Evaluate your current quality of life.

7 Has sensitivity changed in the nipple/areola complex, increased/decreased? - On a scale from 1 to 5

(1: very little/least amount,

5: A lot/largest amount)

8 Was there a significant amount of swelling in and around the breast area?

9 Are you less likely to show yourself in public?

10 Has your self-confidence level changed due to the surgery? 


\section{RESULTS}

The mean age of the 50 patients studied was 48.7 years (range, $28-65$ years). $16 \%$ of the patients have diabetes mellitus and $28 \%$ are hypertensive. As regard the tumor location, it was in the right breast in 28 patients and left breast in 22 patients, $70 \%$ of the tumors were located in the upper outer breast quadrant, $76 \%$ of patients had clinically positive axillary lymph nodes, and $70 \%$ of the patients had neoadjuvant chemotherapy to reduce the mass size (Table 2) \& (Fig. 4).

Table (2): Baseline data of enrolled women.

\begin{tabular}{ll}
\hline & $\mathrm{N}=50$ \\
\hline Age range (years): & $28-65$ \\
$\quad$ Mean & 48.7 \\
Smokers & $0(0 \%)$ \\
Obesity (BMI > 30) & $9(18 \%)$ \\
Hypertension & $14(28 \%)$ \\
Diabetes mellitus & $8(16 \%)$ \\
Affected breast: & \\
$\quad$ Right & $28(56 \%)$ \\
$\quad$ Left & $22(44 \%)$ \\
Site of the mass: & \\
$\quad$ Upper outer quadrant & $35(70 \%)$ \\
Upper inner quadrant & $8(16 \%)$ \\
$\quad$ Upper pole (12 o'clock) & $7(14 \%)$ \\
Distance from the nipple: & $1.5-11.5 \mathrm{~cm}$ \\
$\quad$ Mean & $6.35 \mathrm{~cm}$ \\
Neoadjuvant therapy & $35(70 \%)$ \\
Clinically suspected axillary LNs & $38(76 \%)$ \\
\hline
\end{tabular}

Data expressed as frequency (percentage).

BMI: Body mass index. LNs: lymph nodes.

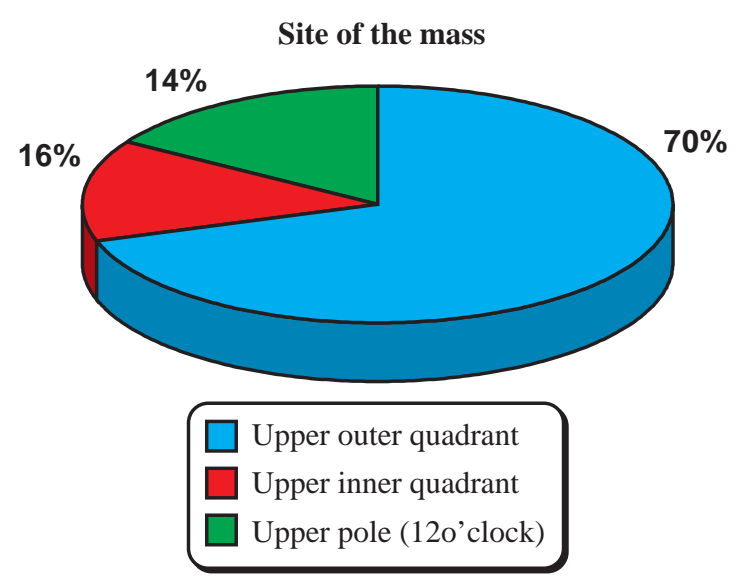

Fig. (4): Site of the mass.

Pre-operative tru-cut biopsies were invasive ductal carcinoma in 44 patients and ductal carcinoma in situ (DCIS) in 6 patients, patient's pathologic stages were as follows: CIS $(n=6), I(n=8)$, IIA ( $n=23)$, IIB $(n=13)$. (Table 3) \& (Fig. 5).
Table (3): Staging of breast cancer among the enrolled women.

\begin{tabular}{cl}
\hline & $\mathrm{N}=50$ \\
\hline Staging: & \\
DCIS & $6(12 \%)$ \\
Stage I & $8(16 \%)$ \\
Stage II A & $23(46 \%)$ \\
Stage II B & $13(26 \%)$ \\
\hline
\end{tabular}

Data expressed as frequency (percentage).

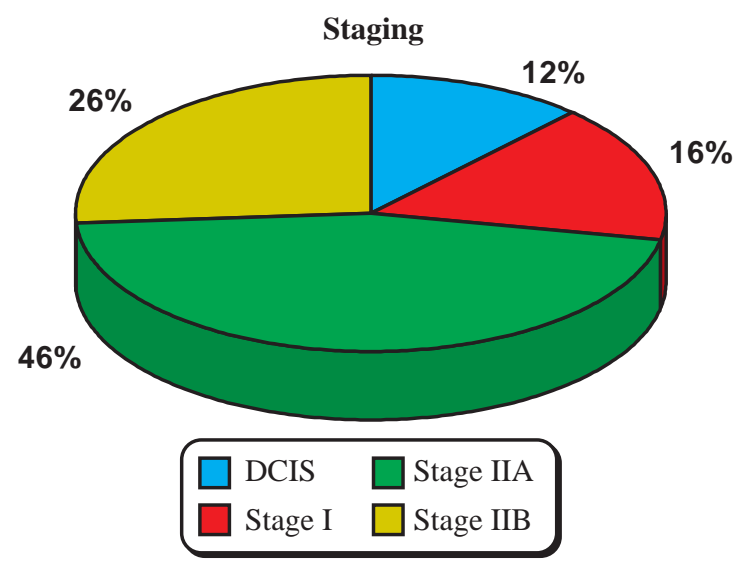

Fig. (5): TNM staging of the patients.

The time taken for the operation was approximately 150-210 minutes, which included the tumor resection with axillary lymph nodes clearance, frozen section pathological evaluation, contralateral breast reduction, and surgical suture. Blood loss, in general, was insignificant (approximately 100$300 \mathrm{cc}$ ). Only $14 \%$ of patients showed one or more positive margins by frozen section in the first time but wider excision was done to get the negative margins all around. (Table 4).

Table (4): Operative data.

\begin{tabular}{ll}
\hline $\begin{array}{l}\text { Operative time: } \\
\quad \text { Mean }\end{array}$ & $150-210$ mints \\
Blood loss & 190 mints \\
Frozen section pathology result: & $100-300 \mathrm{cc}$ \\
$\quad$-ve margins & $43(86 \%)$ \\
$\quad$ +ve margins and wider excision was done & $7(14 \%)$ \\
\hline Data expressed as frequency (percentage). &
\end{tabular}

Hospital stay was only 1 day for all patients, the drainage volume was up to $100 \mathrm{ccs}$ and the drain was removed in the second or the third day. (Table 5).

Table (5): Post-operative data.

\begin{tabular}{ll}
\hline Hospital stay & 1 day \\
Drainage volume & $80-100 \mathrm{cc}$ \\
Drain removal & $2-3$ days \\
\hline
\end{tabular}


Bilateral inferior pedicle mammoplasty was done for the 50 patients (in the first follow-up, 2 patients developed wound seroma and another 4 patients developed wound dehiscence, which required just frequent dressing and an antibiotic). In the second follow-up $96 \%$ of patients had good symmetry and $4 \%$ had fair symmetry, of these patients $98 \%$ of the patients were highly satisfied with the aesthetic outcome and $2 \%$ had fair satisfaction.

Evaluation of the patients in the third and last postoperative visit revealed that $94 \%$ of patients were highly satisfied with the aesthetic outcome and $90 \%$ of the patients had good symmetry. The percentage of symmetry was reduced mostly due to the effect of radiotherapy on the breast from which the tumor was resected.

We didn't encounter any recurrence during the 18 months of follow-up. (Table 6) \& (Figs. 6,7,8).

Table (6): Follow-up of patients.

\begin{tabular}{|c|c|}
\hline $\begin{array}{l}\text { Bilateral inferior } \\
\text { Pedicle mammoplasty }\end{array}$ & $\mathrm{N}=50$ \\
\hline \multicolumn{2}{|l|}{$1^{\text {st }}$ follow-up: } \\
\hline No complications & $44(88 \%)$ \\
\hline Seroma & $2(4 \%)$ \\
\hline Wound dehiscence & $4(8 \%)$ \\
\hline NAC necrosis & $0(0 \%)$ \\
\hline \multicolumn{2}{|l|}{$2^{\text {nd }}$ follow-up (1 month): } \\
\hline \multicolumn{2}{|l|}{ Symmetry } \\
\hline Good & $48(96 \%)$ \\
\hline Fair & $2(4 \%)$ \\
\hline Poor & $0(0 \%)$ \\
\hline \multicolumn{2}{|l|}{ Satisfaction } \\
\hline Good & $49(98 \%)$ \\
\hline Fair & $1(2 \%)$ \\
\hline Poor & $0(0 \%)$ \\
\hline \multicolumn{2}{|l|}{$3^{\text {rd }}$ follow-up (18 months): } \\
\hline \multicolumn{2}{|l|}{ Symmetry } \\
\hline Good & $45(90 \%)$ \\
\hline Fair & $5(10 \%)$ \\
\hline Poor & $0(0 \%)$ \\
\hline \multicolumn{2}{|l|}{ Satisfaction } \\
\hline Good & $47(94 \%)$ \\
\hline Fair & $3(6 \%)$ \\
\hline Poor & $0(0 \%)$ \\
\hline Recurrence & $0(0 \%)$ \\
\hline
\end{tabular}

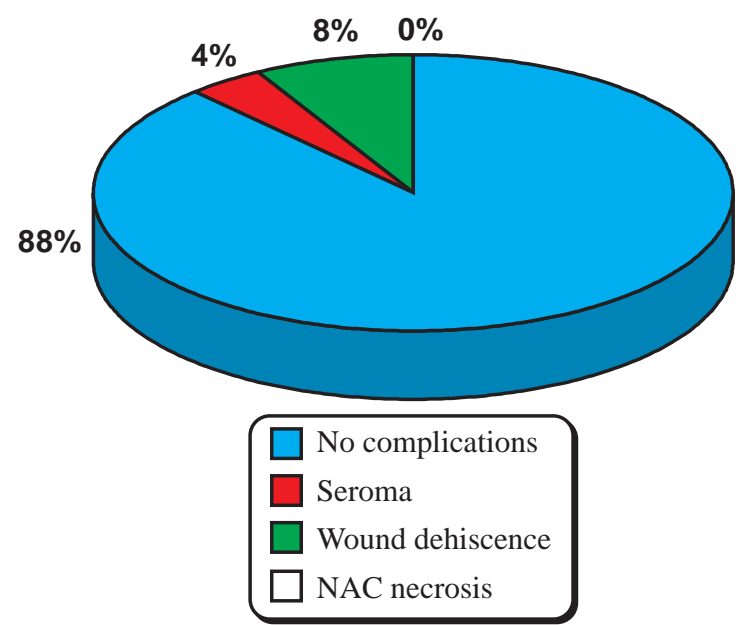

Fig. (6): Post-operative.

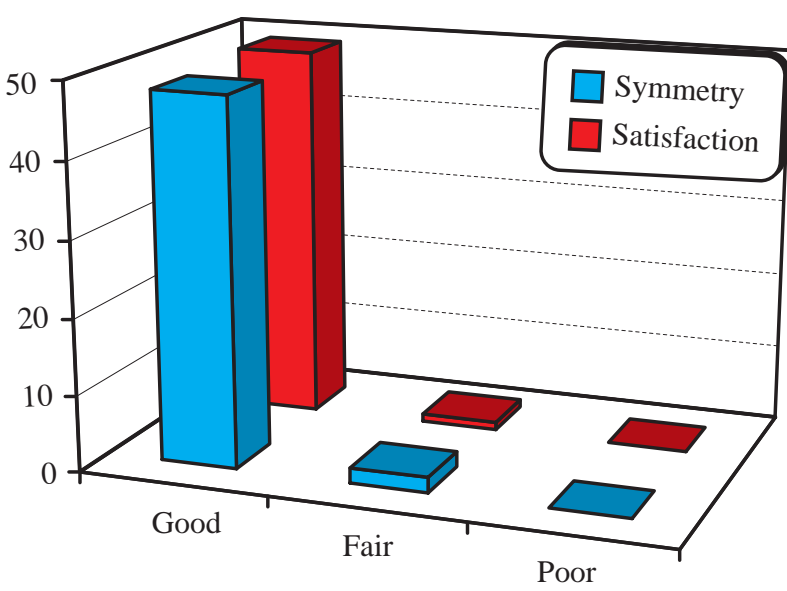

Fig. (7): $2^{\text {nd }}$ follow-up.

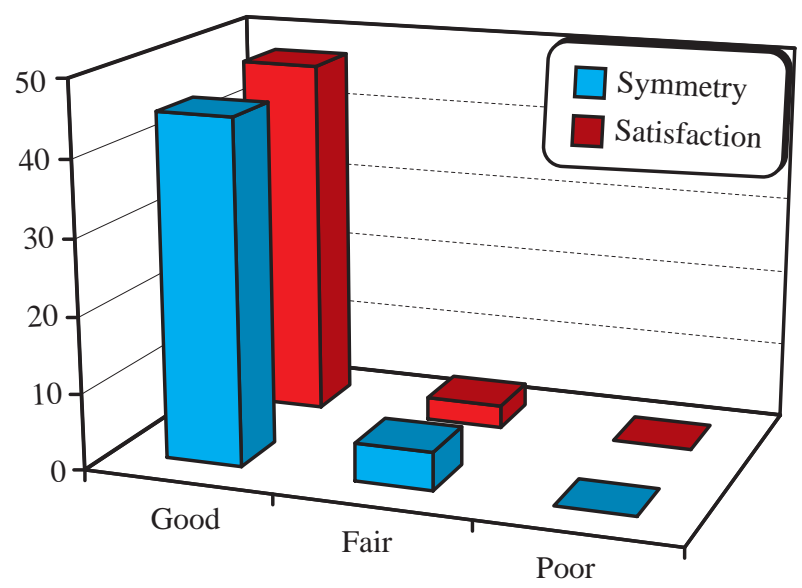

Fig. (8): $3^{\text {rd }}$ follow-up.

\section{DISCUSSION}

The combination of breast conservative surgery and post-operative radiotherapy has been accepted as the standard treatment for early breast cancer cases (stages: In situ, I, IIA, and IIB). 
The increasing female awareness about breast cancer and the developed screening programs together with the developed treatment modalities as adjuvant chemotherapy and hormonal therapy resulted in an increase in both patients' survival and disease-free survival [11].

Surgical treatment of breast cancer has evolved mainly to cover the most important three areas: Oncological safety, patient smooth recovery, and the best aesthetic outcome [12].

As the standard BCS resulted in poor cosmetic outcome even with limited resected volumethis led to the development of the OPS which combines both the oncological resection techniques together with plastic techniques to get more acceptable cosmetic results without affecting the oncological safety and allows surgeons for much more volume to be resected so OPS has gained widespread acceptance in the recent decade [11].

Another important factor in determining the cosmetic outcome is tumor location. Although the upper outer quadrant of the breast is a favorable location for large volume excisions, there are less favorable locations such as the upper pole or upper inner quadrants of the breast often results in breast deformity [13].

The developed techniques of OPS and the addition of efforts from both oncosurgeons and plastic surgeons led to the more aesthetic accepted operations as the therapeutic mammoplasty techniques which have a much accepted aesthetic outcome and the great value of bilateral symmetrization without affecting the oncological outcome [12,13].

In this study, we report 50 female patients hadtherapeutic mammoplasty, and the aesthetic outcome was highly satisfactory. In general, we have offered OPS when the expected resected breast tissue is relatively high and BCS would likely result in a low cosmetic outcome Also, OPS is offered when there is a significant risk of margin involvement, such as, in CIS as it allows resection of larger masses with wider safety margins [14].

In our study, the mean age was 48.7 years (2865 years) which iscomparable to the study done by Emirolgu et al., [12] (50 years) who did therapeutic mammoplasty also, but it's relatively lower than the mean age in the studies carried out by Rose et al., [15] (53 years), Tenofsky et al., [16], (60.9) years and Eichlier et al., [10] (53.3) years who did unilateral different oncoplastic techniques or simple BCS. This was expected as the younger women have much interest in breast symmetry and aesthetic outcomes. The younger age of the included patients in this study increased the aestheticdemand of the patients which was a more challenging goal in our study.

In our study, the tumor site in the breast was 35,8 and 7 in the upper outer quadrant, upper inner quadrant, and the upper pole respectively. The tumor size ranged from $0.7 \mathrm{~cm}$ to $3.4 \mathrm{~cm}$ and its distance from the nipple ranged from 1.5 to 11.5 $\mathrm{cm}$ with a mean distance of $6.35 \mathrm{~cm}$. This was higher than those of patients included in the study carried out by Chen [17] (2-6cm from the nipple). This shows the greater variability in site, size, and distance of the tumor from the NAC that can be treated by therapeutic mammoplasty which approves the wide role of therapeutic mammoplasty in treating a wide spectrum of breast cancers.

As regards the operative time and intraoperative blood loss, in our study, the operative time was longer and blood loss was more than unilateral OPS with meantime: $3.2 \mathrm{~h}$ and $100-300 \mathrm{ml}$ blood loss (average: $203.33 \mathrm{ml}$ ). Ogawa [18] in his study of 18 patients reported a mean operative time of 3h (range: 188-191min) in the unilateral round block OPS technique group. Emirolgu et al., [12] in their study of 82 patients reported that the average operative time in the reduction mammoplasty group was $2.5 \mathrm{~h}$ (range: $80-190 \mathrm{~min}$ ). In our study, we found that the mean hospital stay was one day.

As regards post-operative complications the overall rate was $12 \%$ (two patients with seroma and four patients with wound dehiscence) three of these patients have diabetes mellitus which may played role in bad healing. All these patients were treated conservatively. This is comparable to the study done by Emirolgu et al., [12] which reported $12.2 \%$ of the 82 patients had complications in form of wound dehiscence, NAC necrosis, seroma, and wound site infection. Losken A. et al., in 2014 reported $16 \%$ complications in the reduction mammoplastytechnique [19] Munhoz AM et al., in 2011 found that the incidence of complication was about $22 \%$ [20]. In the Ogawa [18] study who did simple OPS (round block technique) on 18 patients, $22.2 \%$ of the developed NAC ischemia. This shows that the vascularity of the nipple, when based on the inferior pedicle, becomes more sufficient.

In our study, we reported the cosmetic outcome according to patient satisfaction and surgeon's satisfaction with the final breast shape and it was 90\% good and $10 \%$ fair Compared to the Zaha et al., [21] study on 40 patients who underwent mod- 
ified round block technique, it was good in $65 \%$, fair in $32.5 \%$ and poor in $2.5 \%$. Denewer et al., [22] in a total of 50 patients that underwent reduction mammoplasty group, the cosmetic outcome was $64 \%$ good, $30 \%$ showed fair results, and $6 \%$ rated the outcome as poor. Also, the results of the study done by Bogusevicius et al., [23] in 2014 found that $87.2 \%$ of patients had good to excellent cosmetic outcomes in patients with locally advanced breast cancer undergoing oncoplastic surgery. The much better results in our study came from the use of bilateral breast reduction techniques and the collaboration between general surgeons and plastic surgeons.

In our study, there was no local recurrence for 18 months. In the Niinikoski et al., [24] study, the local recurrence rate during a median of 75 months follow-up was $2.3 \%$. Romics et al., [25] reported a recurrence rate of $2.7 \%$ during a median followup of 30 months and Clough et al., [26] reported a local recurrence rate of $2.2 \%$ during a median follow-up of 55 months.

Therefore, we do believe that OPS surgery is safe even in larger breast cancers. This is consistent with the results from prior studies as previously mentioned. Furthermore, we did evaluate the short oncological safety of OPS, as we reported zero local recurrence rates within 18 months followup, a more long-term follow-up is planned.

\section{Conclusion:}

In conclusion progress in the breast cancer surgery over the last years and the new era of oncoplastic techniques has led to more satisfaction and less psychological impact on the operated females without affecting the main aim of oncologic control. That's why therapeutic mammoplasty should be considered as the main therapeutic line for early breast cancer whenever feasible.

\section{REFERENCES}

1- Campbell E.J. and Romics L.: Oncological safety and cosmetic outcomes in oncoplastic breast conservation surgery, a review of the best level of evidence literature. Breast Cancer: Targets and Therapy, 9: 521, 2017.

2- Bohon C.: Cancer recognition and screening for common breast disorders and malignancy. Obstet. Gynecol. Clin., 44: 257-270, 2017.

3- Ahmed A.R.: HER2 expression is a strong independent predictor of nodal metastasis in breast cancer. J. Egypt Natl. Cancer Institute, 28: 219-227, 2016.

4- Kaufman C.S.: Increasing Role of Oncoplastic Surgery for Breast Cancer. Curr. Oncol. Rep., 21 (12): 111. Published 2019 Dec 14. doi:10.1007/s11912-019-0860-9, 2019.
5- Silverstein M.J., Lagios M.D., Recht A., et al.: Imagedetected breast cancer: State of the art diagnosis and treatment. Journal of the American College of Surgeons, Oct. 1; 201 (4): 586-97, 2005.

6- Douvetzemis S.E. and Kovacs T.: Concept, Principles and Indication of Oncoplastic Breast Surgery: Fashion or Necessity. In Oncoplastic Breast Surgery Techniques for the General Surgeon (pp. 1-33). Springer, Cham., 2020.

7- Shaikh K., Krishnan S. and Thanki R.: Artificial Intelligence in Breast Cancer Early Detection and Diagnosis. Springer International Publishing AG, Dec 4, 2020.

8- Piper M., Peled A.W. and Sbitany H.: Oncoplastic breast surgery: Current strategies. Gland Surg., 4: 154, 2015.

9- McIntosh J. and O'Donoghue J.M.: Therapeutic mammaplasty-a systematic review of the evidence. European Journal of Surgical Oncology (EJSO), Mar 1; 38 (3): 196202, 2012.

10- Eichlier C., Kolsch M., Sauerwald A., Bach A., Gluz O. and Warm M.: Lumpectomy versus mastopexy? a postsurgery patient survey. Anticancer Res., 33: 731-736, 2013.

11- Najafi M., Salmon R. and Kaviani A.: Oncological outcome of oncoplastic breast surgery: A review of the literature. Archives of Breast Cancer, Feb. 28: 5-14, 2015.

12- Emiroglu M., Karaali C., Salimoglu S., Sert I. and Aydın C.: Oncoplastic reduction mammoplasty for breast cancer in women with macromastia: Long term aesthetic, functional and satisfaction outcomes. Contemp. Oncol., 20: 256, 2016.

13- Dixon J.M., editor. Breast Surgery: Companion to Specialist Surgical Practice. Elsevier Health Sciences, Jun 22, 2013.

14- Jeevan R., Cromwell D.A., Trivella M., et al.: Reoperation rates after breast conserving surgery for breast cancer among women in England: Retrospective study of hospital episode statistics. BMJ, Jul 12; 345, 2012.

15- Rose M., Manjer J., Ringberg A. and Henry Svensson: Surgical strategy, methods of reconstruction, surgical margins and postoperative complications in oncoplastic breast surgery. Eur. J. Plast. Surg., 37: 205-214, 2014.

16- Tenofsky P.L., Dowell P., Topalovski T. and Helmer S.D.: Surgical, oncologic, and cosmetic differences between oncoplastic and nononcoplastic breast conserving surgery in breast cancer patients. Am. J. Surg., 207: 398-402, 2014.

17- Chen D.R.: An optimized technique for all quadrant oncoplasty in women with small-to medium-sized breasts. Eur. Rev. Med. Pharmacol. Sci., 18: 1748-1754, 2014.

18- Ogawa T.: Usefulness of breast-conserving surgery using the round block technique or modified round block technique in Japanese females. Asian J. Surg., 37: 8-14, 2014.

19- Losken A., Dugal C.S., Styblo T.M. and Carlson G.W.: A meta-analysis comparing breast conservation therapy alone to the oncoplastic technique. Annals of Plastic Surgery, Feb 1; 72 (2): 145-9, 2014.

20- Munhoz A.M., Aldrighi C.M., Montag E., et al.: Outcome analysis of immediate and delayed conservative breast surgery reconstruction with mastopexy and reduction mammaplasty techniques. Annals of Plastic Surgery, Sep. 1; 67 (3): 220-5, 2011. 
21- Zaha H., Onomura M. and Unesoko M.: A new scarless oncoplastic breastconserving surgery: Modified round block technique. Breast, 22: 1184-1188, 2013.

22- Denewer A., Shahatto F., Elnahas W., Farouk O., Roshdy S. and Khater A.: Therapeutic reduction mammoplasty in large-breasted women with cancer using superior and superomedial pedicles. Breast Cancer, 4: 167, 2012.

23- Bogusevicius A., Cepuliene D. and Sepetauskiene E.: The integrated evaluation of the results of oncoplastic surgery for locally advanced breast cancer. The Breast Journal, Jan. 20 (1): 53-60, 2014.

24- Niinikoski L., Leidenius M.H., Vaara P., Voynov A.,
Heikkilä P. and Mattson J.: Resection margins and local recurrences in breast cancer: Comparison between conventional and oncoplastic breast conserving surgery. Eur. J. Surg. Oncol., 45: 976-982, 2019.

25- Romics L., Macaskill E.J., Fernandez T., Simpson L., Morrow E. and Pitsinis V.: A population-based audit of surgical practice and outcomes of oncoplastic breast conservations in Scotland - an analysis of 589 patients. Eur. J. Surg. Oncol., 44: 939-944, 2018.

26- Clough K.B., van la Parra R.F., Thygesen H.H., Levy E., Russ E. and Halabi N.M.: Long-term results after oncoplastic surgery for breast cancer: A 10-year follow-up. Ann. Surg., 268: 165-171, 2018. 